\title{
Le problème de la régulation chez Durkheim
}

\section{Philippe Steiner}

\section{OpenEdition}

\section{Journals}

Édition électronique

URL : http://journals.openedition.org/ress/3382

DOI : $10.4000 /$ ress.3382

ISSN : 1663-4446

\section{Éditeur}

Librairie Droz

\section{Édition imprimée}

Date de publication : 1 mars 2004

Pagination : 315-320

ISBN : 2-600-00941-8

ISSN : 0048-8046

Référence électronique

Philippe Steiner, "Le problème de la régulation chez Durkheim », Revue européenne des sciences sociales [En ligne], XLII-129 | 2004, mis en ligne le 25 janvier 2016, consulté le 10 décembre 2020. URL : http://journals.openedition.org/ress/3382 ; DOI : https://doi.org/10.4000/ress.3382

Ce document a été généré automatiquement le 10 décembre 2020.

(C) Librairie Droz 


\title{
Le problème de la régulation chez Durkheim
}

\author{
Philippe Steiner
}

1 On doit à l'ouvrage que Philippe Besnard a consacré à l'anomie - L'anomie, ses usages et ses fonctions dans la discipline sociologique - une claire démonstration concernant deux points décisifs dans la théorie de la socialisation telle qu'on la trouve exposée dans deux des principaux ouvrages de Durkheim: De la division du travail social et, surtout, Le suicide. En premier lieu vient la démonstration lumineuse de la différence entre le processus d'intégration sociale et le processus de régulation sociale; en deuxième lieu figure une définition limpide de l'intégration sociale. Le présent texte commencera donc par rappeler ces deux acquis fondamentaux ( $\$ 1)$; mais ce qui nous intéresse plus particulièrement c'est d'essayer d'approfondir un troisième point, laissé en partie en suspend par l'étude de Besnard, en examinant le processus de régulation dont l'anomie n'est qu'une des conséquences possibles de son mauvais fonctionnement. Nous tâcherons donc de rassembler les arguments donnant consistance à la notion de régulation dans la sociologie durkheimienne, puis de considérer l'effervescence sociale qui lui est étroitement liée (\$2).

\section{Intégration et régulation chez Durkheim selon Besnard}

2 Rappelons tout d'abord la nature des acquis dus au travail de Besnard à propos de la différence entre l'intégration sociale et la régulation sociale. Au delà de l'exégèse fine du texte de Durkheim (Besnard 1987: 70-4), c'est en s'appuyant sur les données de Durkheim que Besnard montre clairement la différence entre les deux processus à propos de l'anomie conjugale. L'intégration étant rapportée à la présence d'enfants, la régulation au statut matrimonial (célibat, mariage,veuvage) (ibid. 74-81), Besnard fait apparaître l'existence d'une courbe en $\mathrm{U}$ montrant qu'une régulation soit trop forte (femmes mariées), soit trop faible (hommes célibataires ou hommes veufs) se traduit par une 
élévation du taux de suicide quel que soit le niveau (satisfaisant ou trop faible) de l'intégration sociale à deux âges (47 et 60 ans).

3 Sur cette base, Besnard rassemble plusieurs passages du Suicide grâce auxquels il élabore une définition générale de l'intégration sociale à partir de l'étude que Durkheim a produite du suicide égoïste, c'est-à-dire en étudiant certaines dimensions de la société religieuse, domestique et politique:

Un groupe social sera dit intégré dans la mesure où ses membres $1 /$ possèdent une conscience commune, partageant les mêmes sentiments, croyances et pratiques (société religieuse, [Durkheim 1897], p. 158-159, 173) ; 2/ sont en interaction les uns avec les autres (société domestique [Durkheim 1897], p. 214);3/ se sentent voués à des buts communs (société politique, [Durkheim 1897], p. 222) (Besnard 1987: 99).

Le résumé est brillant et, pensons nous, définitif en matière de la théorie durkheimienne de l'intégration sociale qui ressort ainsi en pleine lumière, surtout après que Besnard ait lumineusement montré la différence entre intégration et régulation. Bersnard ne manque pas de citer le passage de Durkheim selon lequel la socialisation ne signifie pas seulement que la société définit les manières de faire, de sentir et de penser des individus, mais aussi qu'elle les règle ${ }^{1}$. Il n'en reste pas moins que le lecteur peut trouver curieuse la façon dont la théorie de la régulation est considérée:

La théorie de la régulation est loin d'être développée de manière aussi systématique. Elle est d'abord, on l'a vu, tronquée en raison du désintérêt de Durkheim à l'égard du fatalisme. Quant à l'anomie, il est bien difficile de la décomposer en éléments comme on a pu l'esquisser à propos de l'intégration (ibid. 99-100).

Besnard note qu'à l'inverse il serait erroné de prendre l'anomie pour un concept unique et il montre que l'on peut décomposer l'anomie - qui est le sujet de son ouvrage, rappelons-le - en différentes catégories selon la durée (anomie aiguë / chronique) ou la tendance d'évolution (anomie régressive / progressive). Mais, dira-t-on, ce faisant, Besnard a déplacé le problème posé, plus qu'il ne l'a réglé. Pourquoi ne pas avoir essayé de reconstruire la théorie de la régulation - et non l'anomie qui n'en est qu'un élément une fois réintroduit le fatalisme que Durkheim minore, ainsi que le fait justement remarquer Besnard? La tentative que Besnard esquisse (ibid. 109-111) s'appuie sur la temporalité pour faire la différence entre l'anomie et le fatalisme selon que le mécanisme générateur est de l'ordre de la crise (temps court) ou de l'ordre du phénomène chronique (temps long). Cette opposition est certes importante pour comprendre l'ouvrage de Durkheim et certains de ses développements, mais on peut se demander s'il est adéquat à l'objet considéré. Les distinctions en termes de temporalité s'appliquent à l'intégration sociale, mais comme Besnard ne les fait pas intervenir pour définir l'intégration sociale, il est difficile d'en tirer une conception qui puisse être homogène à celle de l'intégration sociale.

6 Nous avons pour notre part tâché d'aller dans un sens un peu différent (Steiner 1992, 1994) en greffant une suggestion sur le travail accompli par Besnard. Nous avons rapporté les trois éléments de l'intégration sociale non pas aux sphères de la vie sociale dans lesquelles Durkheim les a inscrites dans Le suicide (le politique, le religieux et la famille), mais aux trois niveaux de l'analyse sociologique durkheimienne avec le niveau morphologique (les manières d'être, ici la structure relationnelle), le niveau physiologique (les manières de faire, ici les croyances et pratiques communes) et, finalement, les représentations (ici les buts et les idéaux communs). 
7 Il est ici peu utile de revenir en général sur cette présentation de l'intégration et de la régulation; nous suggérons plutôt d'en considérer un élément particulier avec l'importance que prend la notion d'idéal dans cette présentation et, par contre coup, la notion d'effervescence sociale qui, comme Besnard l'a bien senti, joue un rôle important pour qui veut comprendre l'économie de l'oeuvre de Durkheim et les phénomènes sociaux qu'il cherchait à expliquer².

\section{Régulation sociale et effervescence: du Suicide aux Formes élémentaires}

En attirant l'attention sur l'effervescence sociale, il est possible de considérer la théorie durkheimienne de la socialisation en la mettant à l'oeuvre tant dans la première phase de ses écrits - c'est-à-dire avant qu'il ne délaisse le programme de travail dont l'esquisse est rappelée dans la préface à la réédition de La division du travail social pour passer à la sociologie de la religion et de l'idéal - que dans la deuxième. Surtout, elle permet de voir à l'oeuvre la réflexion de Durkheim sur la régulation sociale alors même que le concept d'anomie - Besnard l'a très clairement montré - disparaît de son vocabulaire sociologique.

Dans les Formes élémentaires, l'effervescence est cette situation particulière de la vie sociale dans laquelle se revivifient ou se créent les idéaux sociaux appartenant à la conscience collective de la tribu. Comme Durkheim y a insisté, ce développement occupe une grande place dans son dispositif de recherches dans la mesure où Durkheim y trouve la réponse à des questions fondamentales de ce qu'il appelle la sociologie de l'idéation collective, savoir : dans quelles configurations sociales se forment les idéaux sociaux? et comment la sociologie, telle qu'il l'entend, peut rendre compte rationnellement de ces configurations et de leurs résultats?

Si maintenant, on rapporte cette configuration sociale - l'effervescence - à la théorie de la socialisation, on peut mettre en lumière quelques traits intéressant la théorie durkheimienne de la régulation. Ce rapprochement se justifie aussi d'un point de vue textuel, une fois que l'on remarque la présence du terme d'effervescence dans Le suicide, phénomène qui joue un rôle surprenant en donnant lieu à des effets de sens contraire sur les taux de suicide: les élevant ici, les diminuant là. Comment expliquer cela?

11 Dans Le suicide, Durkheim emploie le terme d'effervescence pour désigner une situation où s'observe une vie sociale plus intense. Le fait qu'une vie sociale plus intense accroît la tendance au suicide contredit la théorie de l'intégration sociale et l'explication du suicide égoïste ${ }^{3}$. Toutefois, cela n'explique pas les sens divergents de la relation entre l'effervescence et le taux de suicide. Durkheim voit l'effervescence politique comme un accroissement des passions politiques ; une appréciation favorable y est attachée en raison de son apport à l'intégration sociale; celle-ci est d'autant plus forte et joue un rôle protecteur d'autant plus important que le niveau de la vie passionnelle est élevé. En réactivant l'importance du but commun politique, en accroissant le niveau passionnel de la vie politique et la fréquence des interactions, l'effervescence élève le degré d'intégration du groupe et, par là, joue un rôle préservatif du suicide; lorsque le phénomène politique de courte période prend fin, la vie politique retombe à son bas niveau et l'atonie de l'intégration sociale du groupe reprend le devant de la scène. 
12 Dans le cas de l'effervescence économique, le déchaînement des passions est propice au suicide anomique, aussi l'effervescence économique débouche sur " le mal de l'infini» qu'est l'anomie. Dans les secteurs industriels et commerciaux, la vie sociale est faite d'une exacerbation des passions économiques, la passion des richesses ou la passion du bienêtre. Les crises économiques ajoutent un surcroît d'activité passionnelle à un niveau qui est déjà très élevé: cela est néfaste, dit Durkheim, car les passions sont déjà affranchies de toutes limites et leur exacerbation ne peut plus être que douloureuse pour les individus. L'élévation brusque du niveau de vie passionnelle ne signifie pas la même chose selon qu'elle affecte la régulation ou l'intégration sociale. Positif pour ce dernier processus, elle ne l'est pas pour le premier parce que le problème y est différent : il s'agit de modérer les passions suivant la place que l'on occupe dans la hiérarchie sociale et non pas d'uniformiser et d'accroître l'intensité des passions qui animent les différents individus appartenant à un même groupe social.

Venons-en maintenant à l'effervescence dans les Formes élémentaires. Lorsque Durkheim (1912: 307s) décrit la vie sociale des tribus australiennes, il prend soin de mettre en évidence la dimension temporelle qui les rythme: aux phases d'éparpillement au cours desquelles la tribu se disperse au grès des impératifs de la recherche de la nourriture font suite des phases de rassemblement. C'est alors l'occasion de renouer le lien social, de retrouver une vie collective intense avec des fêtes et des cérémonies religieuses dont les moments les plus forts sont étudiés en tant que périodes d'effervescence sociale. L'effervescence sociale signifie que les règles de la vie commune sont momentanément suspendues et c'est à cette condition que le milieu effervescent peut se dérouler et aboutir au résultat qui est le sien. Le caractère dérégulé, orgiaque, de l'assemblée effervescente est clairement indiqué par la terminologie durkheimienne ${ }^{4}$ : la dérégulation affecte des pans importants de la vie sociale (notamment sexuelle) en élevant le niveau de vie passionnel après que celui-ci se soit affaibli pendant la période de dispersion de la tribu pour des raisons économiques. Si l'excitation exceptionnelle des passions entraîne une dérégulation exceptionnelle elle offre un résultat lui aussi tout à fait exceptionnel : défaisant momentanément les contraintes qui assurent la régulation sociale, le milieu effervescent donne un plus haut degré d'intégration sociale, en élevant le niveau de la vie passionnelle immédiate et en fournissant un idéal grâce auquel, dans la durée, l'intégration et la régulation sortent renforcées.

14 Cette conclusion est conforme à la façon dont Durkheim apprécie les transformations des doctrines pédagogiques à la Renaissance (Durkheim 1904-5: 210-211, 218-220; Besnard 1993). L'appréciation très élogieuse faite de la pensée de Rabelais est conduite en des termes identiques à ceux que Durkheim employait jadis pour caractériser négativement la situation engendrant l'anomie. Les idées rabelaisiennes sont l'archétype d'un rejet de toute réglementation, de toute discipline, de tout frein, de toute borne. La société est alors exubérante, recèle une pléthore de vie, elle accomplit une révolution en mettant au jour de nouveaux idéaux pédagogiques, après un Moyen Age riche et qui n'avait pas lui non plus ignoré ce phénomène à la période des Croisades (ibid. 79-81, 88). Avec la Renaissance, à l'origine de la société moderne donc, à l'occasion d'une institution sociale d'une grande importance (le système scolaire), Durkheim retrouve les accents qui glorifient la dérégulation créatrice d'idéaux. Dans le cadre des tribus australiennes, comme dans celui de l'institution scolaire à la Renaissance, l'effervescence sociale est jugée positivement en renvoyant à la création d'idéaux propres à renforcer la cohésion sociale. 


\section{Conclusion}

15 manière à ne pas mettre en avant les conséquences d'une régulation trop forte alors même qu'il appelait à un renforcement de la réglementation sociale, notamment en matière économique, il ne semble pas avoir reculé devant l'effervescence, forme exacerbée de dérégulation sociale. La raison, selon nous, tient au fait que si le thème de l'anomie disparait en tant que forme de l'affaiblissement de la régulation, cette disparition laisse la place à une forme positive de régulation affaiblie avec l'effervescence, laquelle permet de renforcer l'intégration sociale sur la longue période.

Sur ce point la démonstration de Besnard est toujours valide quant à la distinction entre les deux processus et le fait que l'action de l'un a un impact sur l'autre - ici au travers d'une relation dynamique. Mais en plaçant l'effervescence sociale à côté de l'anomie, puis à la place de cette dernière, on donne toute sa place à la question de l'idéal, question à laquelle Durkheim attachait beaucoup d'importance. On est ainsi conduit à penser que Durkheim n'est ni seulement, ni principalement, le théoricien de l'intégration sociale, car il est tout autant le théoricien de la régulation sociale par l'intermédiaire, non de l'anomie, mais de l'idéal, c'est-àdire du monde des valeurs et de la création de ces dernières, un monde bien différent du positivisme étroit que, trop souvent, on associe au nom du grand sociologue français.

\section{BIBLIOGRAPHIE}

Besnard Ph. (1973), «Durkheim et les femmes ou le Suicide inachevé», Revue française de sociologie, 14(1) : 27-61.

- (1987), L'anomie, ses usages et ses fonctions dans la discipline sociologique depuis Durkheim, Paris : Presses universitaires de France.

- (1993), «De la datation des cours pédagogiques de Durkheim à la recherche du thème dominant de

son oeuvre», in F. Cardi \& J. Plantier (eds.), Durkheim sociologue de l'éducation, Paris : L'Harmattan: 120-130.

Cherkaoui M. (1998), La naissance d'une science sociale. La sociologie selon Durkheim, Genève: Droz.

Durkheim É. (1897), Le suicide. Étude de sociologie, Paris : Presses universitaires de France, 1983.

- (1912), Les formes élémentaires de la vie religieuse, Paris : Presses universitaires de France, 1968.

Paoletti G. (2002), « Il quadrato di Durkheim. La definizione del legame sociale e suoi critici», in M.

Revue européenne des sciences sociales, XLII-129 | 2004 
Rosati \& A. Santambrogio (éd.), Émile Durkheim, contributi ad una rilettura critica, Roma:

Meltemi : 235-261.

Steiner Ph. (1992), «Le fait social économique chez Durkheim», Revue française de sociologie,

33(4) : 641-661.

- (1994), La sociologie de Durkheim, Paris : La découverte.

- (2004), Économie et religion. Principes de la sociologie économique durkheimienne, à paraître.

\section{NOTES}

1. La formulation de Durkheim est la suivante: «Mais la société n'est pas seulement un objet qui attire à soi, avec une intensité inégale, les sentiments et l'activité des individus. Elle est aussi un pouvoir qui les règle» (Durkheim 1897: 264).

2. A cet égard, il est intéressant de considérer les remarques de Giovanni Paoletti (2001), notamment

lorsqu'il introduit, ainsi que Mohamed Cherkaoui (1998), la question de la justice dans la construction durkheimienne. En effet, la justice renvoie à un phénomène essentiel de la sociologie

de Durkheim, d'une part parce qu'elle définit une modalité de l'anomie dans De la division du travail social, mais aussi parce qu'elle met en jeu le mécanisme de formation des idéaux, question qui préoccupe tout particulièrement Durkheim, ainsi qu'on le voit dans sa lettre à Célestin Bouglé à propos du compte rendu des Formes élémentaires, ou bien dans son article de 1913 «Jugements de faits et jugements de valeur».

3. Besnard (1973) a clairement mis ce point en évidence dans son article consacré à montrer l'inachèvement de l'analyse de la place des femmes dans Le suicide.

4. La description faite par Durkheim passe par des termes sans équivoques sur ce point : il est question

de «passions vives et affranchies de tout contrôle», de «tumultes», de « relations sexuelles en dehors des règles qui président au commerce sexuel», d' " incestes», de « délire et nervosité pathologique», etc.

\section{AUTEUR}

\section{PHILIPPE STEINER}

Université Lille III \& IRIS, Université de Paris-IX

Philippe.Steiner@dauphine.fr 